\title{
Progress in Crosswell Induction Imaging for EOR: Field System Design and Field Testing
}

\author{
B. A. Kirkendall, J. P. Lewis, S. L. Hunter, and P. E. Harben
}

March 4, 1999

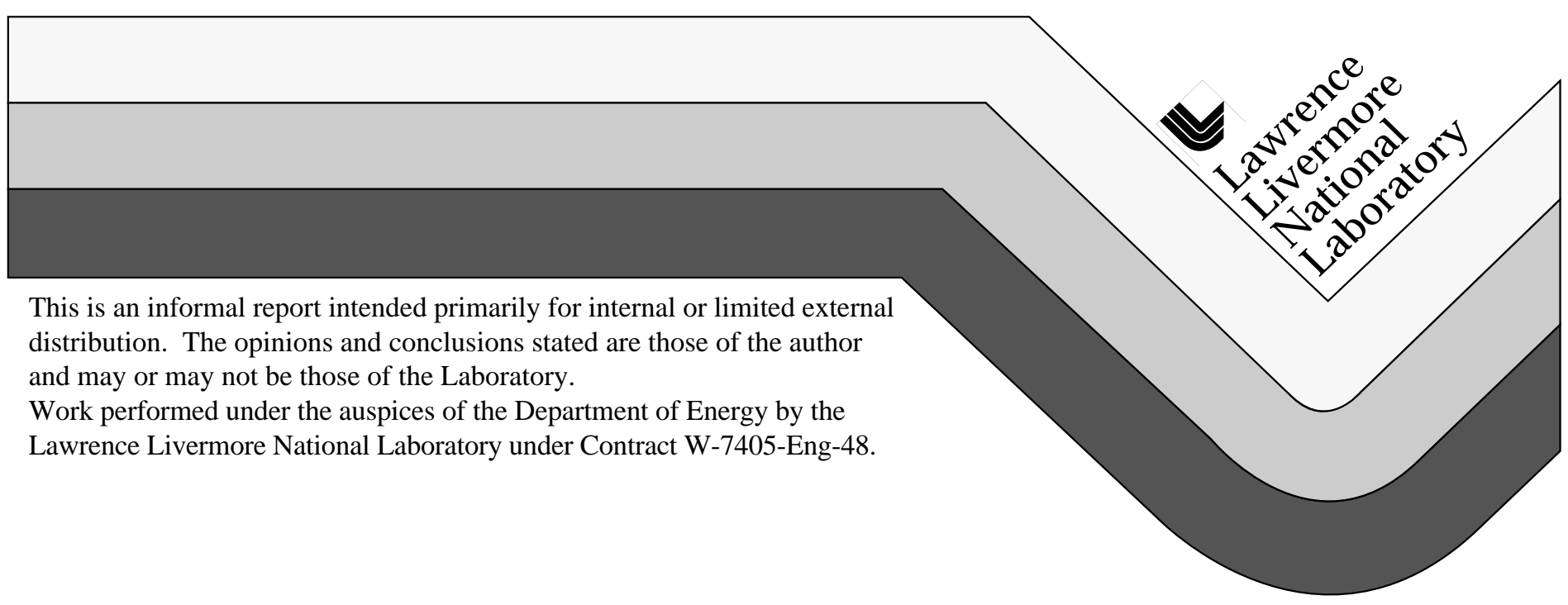




\section{DISCLAIMER}

This document was prepared as an account of work sponsored by an agency of the United States Government. Neither the United States Government nor the University of California nor any of their employees, makes any warranty, express or implied, or assumes any legal liability or responsibility for the accuracy, completeness, or usefulness of any information, apparatus, product, or process disclosed, or represents that its use would not infringe privately owned rights. Reference herein to any specific commercial product, process, or service by trade name, trademark, manufacturer, or otherwise, does not necessarily constitute or imply its endorsement, recommendation, or favoring by the United States Government or the University of California. The views and opinions of authors expressed herein do not necessarily state or reflect those of the United States Government or the University of California, and shall not be used for advertising or product endorsement purposes.

This report has been reproduced directly from the best available copy.

Available to DOE and DOE contractors from the Office of Scientific and Technical Information

$$
\text { P.O. Box 62, Oak Ridge, TN } 37831
$$

Prices available from (615) 576-8401, FTS 626-8401

Available to the public from the National Technical Information Service

U.S. Department of Commerce 5285 Port Royal Rd. Springfield, VA 22161 


\title{
Progress in Crosswell Induction Imaging for EOR: Field System Design and Field Testing
}

\author{
B. A. Kirkendall, J. P. Lewis, S. L. Hunter, and P. E. Harben
}

\begin{abstract}
At Lawrence Livermore National Laboratory (LLNL), we are continuing our effort to develop improved crosswell low-frequency electromagnetic imaging techniques, which are used to map in situ steamflood and waterflood movement during enhanced oil recovery (EOR) operations. Toward this effort, we procured two new borehole-logging field vehicles, and developed and integrated new crosswell electromagnetic transmitter and receiver data acquisition and control systems into these vehicles. We tested this new acquisition system by conducting a suite of background measurements and repeatability experiments at the Richmond Field Station in Richmond, California. Repeatability of a given scan in which the receiver was fixed and the transmitter position was varied over $60 \mathrm{~m}$ in $0.2-\mathrm{m}$ increments resulted in amplitude differences of less than $0.6 \%$ and phase differences of less than $0.54 \mathrm{deg}$. Forward modeling produced a resistivity map fully consistent with well log data from the Richmond Field Station. In addition, modeling results suggest (1) that residual high-conductivity saltwater, injected in 1993 and pumped out in 1995, is present at the site and (2) that it has diffused outward from the original target strata. To develop crosswell electromagnetic imaging into a viable commercial product, our future research must be a two-fold approach: (1) improved quantification of system noise through experiments such as ferromagnetic core characterization as a function of temperature, and (2) development of procedures and codes to account for steel-cased hole scenarios.
\end{abstract}

\section{Introduction}

The United States has extracted about 160-billion barrels of oil from its petroleum reserves over the past 130 years. Estimates indicate that this is only about one-third of the total U.S. reserves, but without enhanced extraction methodology, the bulk of these reserves will remain locked in the reservoir formations. To increase the fraction of reservoir oil available for extraction, U.S. oil companies must apply methods of enhanced oil recovery (EOR) to more and more fields.

In California, for example, where the oil is mostly a heavy viscous type, $55 \%$ of the oil currently produced comes from injecting steam, water, or gas into the reservoir to increase production by lowering the viscosity of this oil through heat and/ or mixing. When production yields dwindle in primary reservoirs, often the first action taken by oil companies is to use hydrofracturing in producing horizons to increase output. Hydrofracturing can boost production by creating porous conduits for otherwise trapped oil to migrate to the producing well. Further recovery methods resort to longer term waterfloods, steamfloods, gasfloods, and chemicalfloods to sweep trapped oil to the producing wells. Although other methods employing in situ burning and microbes are in the early stages of development, waterfloods and steamfloods are the predominant means used at present.

It is of great economic importance to know where the steam or waterflood front is going during the EOR process. Channeling, bypass, and breakthrough into a nonproducing horizon can and often do occur. Since many steam and waterfloods operate for years at a time, companies can save money when a sweep problem is discovered early. Although sophisticated reservoir simulators 
are often used to predict the migration of a steam or waterflood through the reservoir, the simulators are limited by (1) the relatively sparse borehole logs available and (2) precise knowledge of the regional geology and in situ rock properties of the reservoir.

Mapping the steam or waterflood with crossborehole methods provides a means to monitor the progress of the EOR process before it reaches another well. The results from front mapping during the flooding process can also be incorporated into the reservoir simulator to further constrain the simulator with measured data from the reservoir.

LLNL pioneered the use of low-frequency cross-borehole electromagnetic imaging to monitor and map the progress of EOR steam and waterfloods. The operating frequencies of the transmitter are in the induction regime and, consequently, the method measures resistivity (or conductivity) contrasts in the reservoir since dielectric permittivity is negligible in the induction regime. The steam or waterflood lowers the resistivity of the formation in the regions that it infiltrates; images taken before, during, and after infiltration are compared to construct a map of the progress of this process. The imaging technique used in this study is depicted in Figure 1. Note that the transmitter and receiver systems are each housed in a specially designed field vehicle.

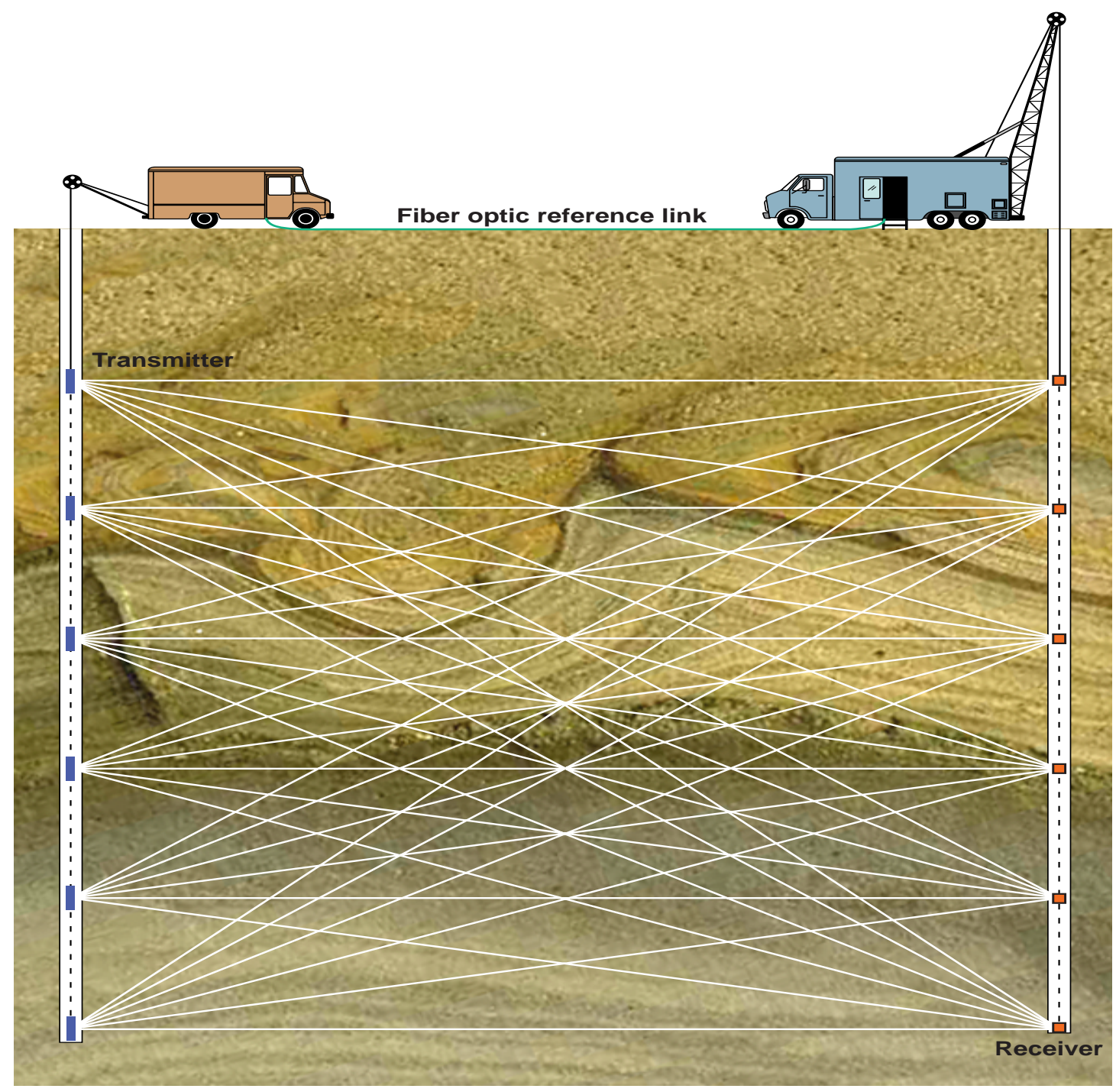

Figure 1. Pictorial representation of the crosswell logging procedure. Although the energy traced between wells does not physically follow raypaths, crosswell processing assumes distribution of such energy along straight conduction pathways. 
The process of imaging an area begins with moving a transmitter over the entire length of the imaging zone so that the receiver can measure the in-phase and out-of-phase components of the signal that penetrate the formation between the two boreholes. These components are measured relative to the transmitter phase using a lock-in amplifier and a fiber-optic reference link to communicate transmitter phase information that is free of inductive coupling - a term that describes a "sneak path" that allows the transmitter signal to reach the receiver through the ground. The next phase in imaging an area consists of lowering the receiver to the next level, where the transmitter is again moved the entire length of the imaging zone so that the receiver can measure the transmitted signal through the formation. The data collected by this imaging process results in a set of measurements with transmitter and receiver positions as indicated by the white lines in Figure 1. An inversion algorithm is later applied to these data to reconstruct the formation resistivity profile between transmitter and receiver boreholes. Note that although the figure depicts straight rays between the transmitter and receiver to indicate the signal path, the true induction field measured by the receiver is influenced by a larger portion of the formation as a modification of a dipole magnetic field.

In this paper, we document the most recent efforts at LLNL to further develop the cross-borehole electromagnetic imaging technology as it applies to monitoring in situ EOR processes. We also describe our continued development of the field data-acquisition system. The first part of this paper details the acquisition system's design and functionality. This is followed by a discussion of the field tests and capability of this new acquisition system. Finally, we present the profiles acquired at the Richmond Field Station (RFS) and compare forward model results with well log and field data; the results of this comparison are another measure of system integrity.

\section{Field Data-Acquisition System}

The new crosswell electromagnetic imaging data-acquisition system is located in two large trucks, both obtained from the U.S. Department of Energy, Nevada Operations Office at the Nevada Test Site. These vehicles were excessed equipment previously used in big-hole logging for nuclear tests. Both vehicles were in nearly new condition, with less than 3000 road miles on the engines. The trucks were refitted as field vehicles to be used specifically for gathering and analyzing crosswell electromagnetic data. The receiver system is set up in a logging truck with a heavy-duty integrated $40-\mathrm{ft}$ boom. The transmitter system is located in a bread truck. The transmitter truck was modified to include a generator, hydraulic system, winch, tailgate-mounted extendable hoist, and a swingable light-duty boom. This section details the modifications to the vehicles and the development and operation of the data acquisition system on each truck.

\section{Receiver Truck}

Figure 2 is a flowchart depicting the data links for the acquisition system located in the receiver truck (Figure 3), which has corresponding fiberoptic links to the transmitter truck, ethernet, voice communication, and dual analog signal receivers.

The TCP/IP protocol in the receiver truck operates under a LabVIEW virtual-data-acquisition instrument on a Windows Pentium PC platform. The signal analog link carrying information about the transmitter coil depth is input into the counter input of one of two National Instruments NB MIO16X E-50 A/D cards; the other card accepts the receiver coil depth directly from the encoder. This depth information is displayed in the receiver truck and is input into the LabVIEW virtual instrument.

The analog receiver coil is essentially a ferromagnetic (mumetal) induction circuit with a dynamic frequency range from $20 \mathrm{~Hz}$ to $100 \mathrm{kHz}$, and is powered by a $12-\mathrm{V}$ dc source. The data from the receiver coil is initially passed through a gain box with the options of signal gain or attenuation, and is then passed into a Stanford Research Systems pre-amplifier. (Electromagnetic energy can become highly attenuated in induction through conductive media, and can often require an amplitude gain to avoid problems with roundoff error and low signal resolution.) Next, the receiver-coil data are passed to a lock-in amplifier, along with the transmitter-current analog signal from the fiberoptic line. The lock-in amplifier uses the transmitter current waveform as the reference signal and detects receiver signals in-phase and out-of-phase; this is an effective method for accurately discriminating low-level signals in a noisy background. The lock-in amplifier is controlled though a GPIB interface on the receiver truck's Pentium PC.

All processing, data storage, and receiver-coil positioning is completed within the receiver truck. 


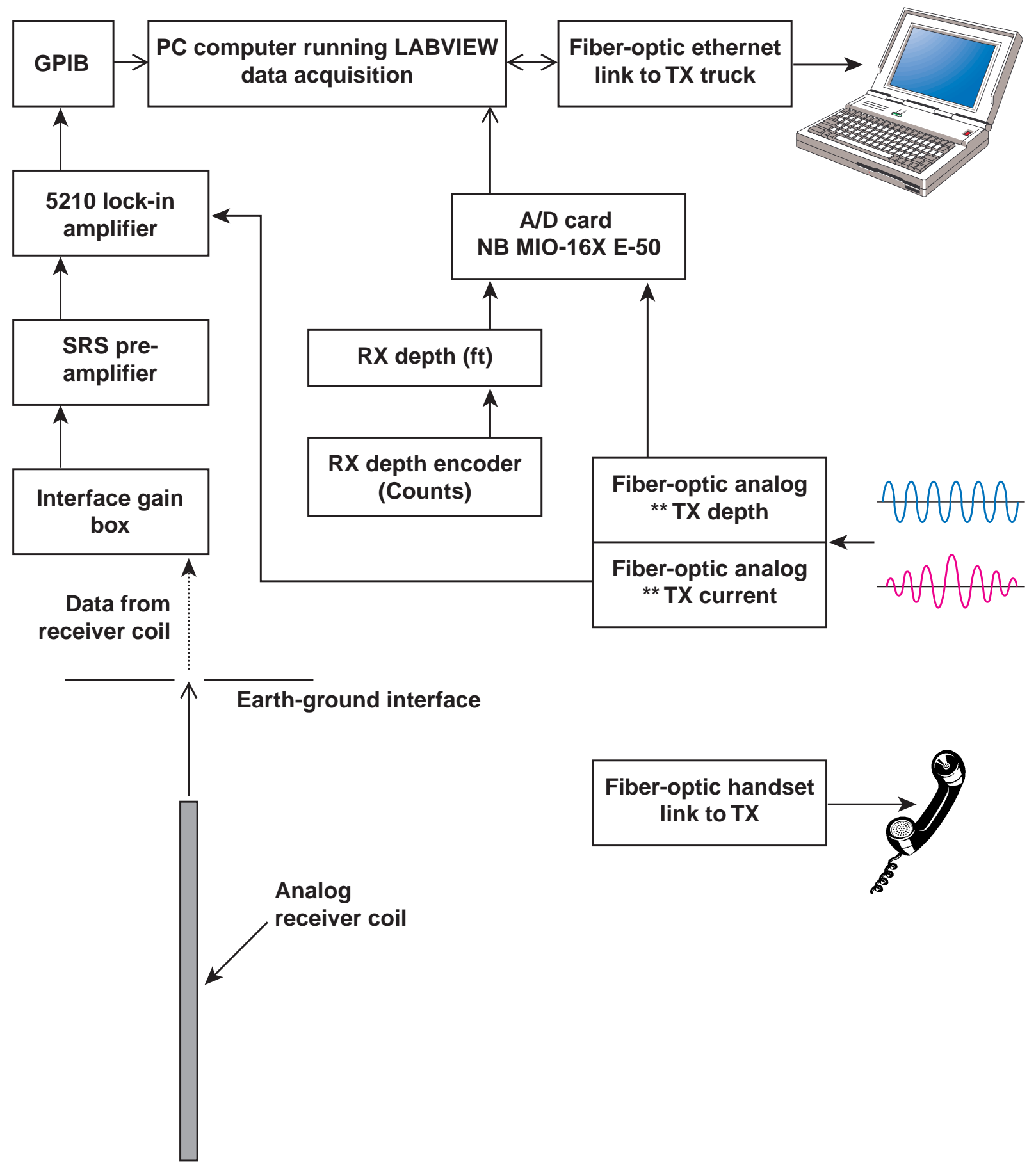

Figure 2. Flowchart of data links in the receiver truck. Fiber-optic cables provide rapid communication using light pulses, thereby eliminating inductive coupling with the acquisition system. 


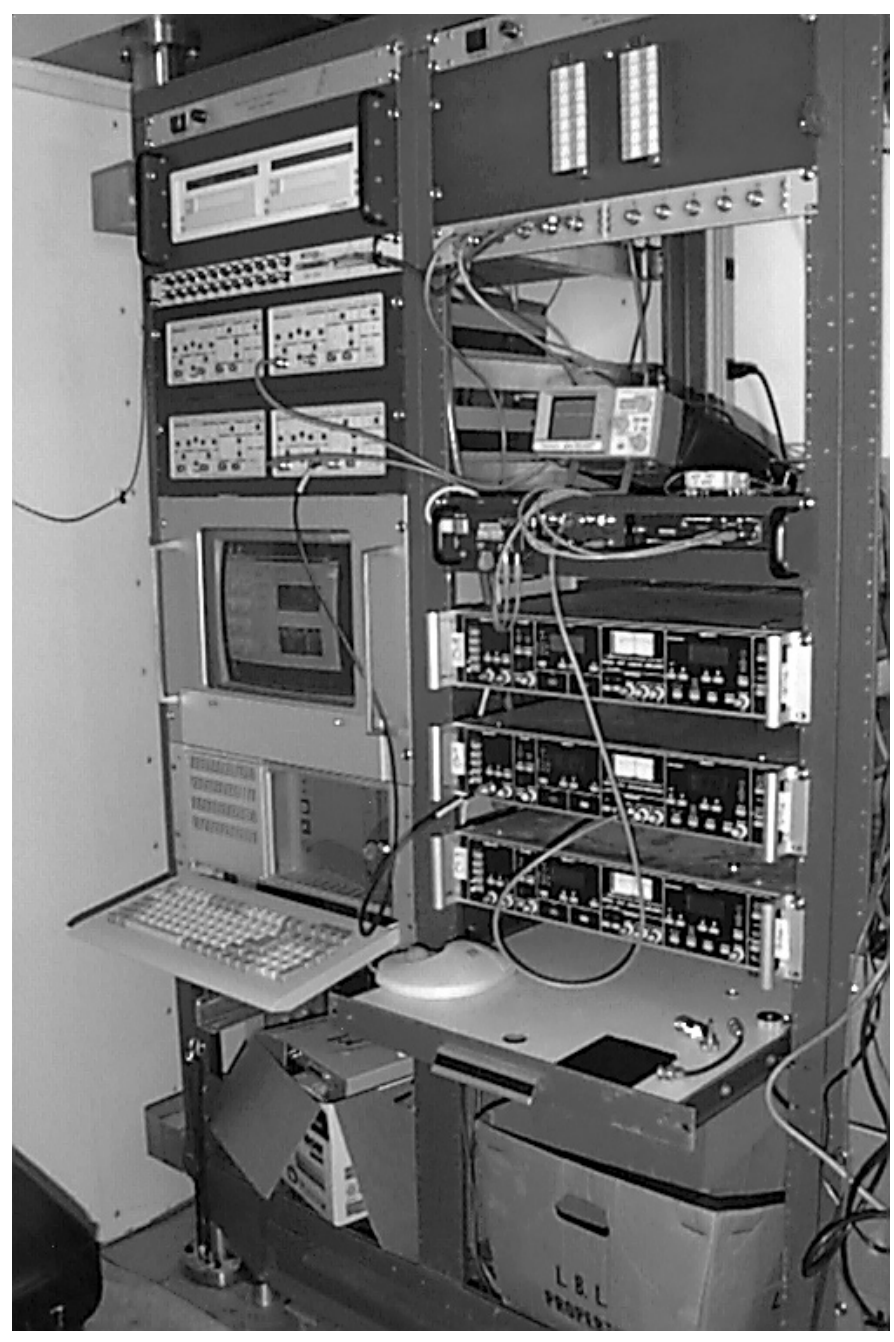

Figure 3. Inside the receiver truck. The fiberoptic chassis contains voice, ethernet, and analog communications. Three lock-in amplifier units are also shown; more than one may be simultaneously used in the presence of excessive signal noise.

In future design changes, we plan to modify the receiver truck so that it can fully operate the transmitter truck, thus making data collection more efficient.

The receiver truck (Figure 4 ) is a heavy duty $6 \times 4$ with a $65,000-1 b$ load rating. The truck was used in logging the large nuclear explosive test holes at the Nevada Test Site. It has an integral boom that provides $40 \mathrm{ft}$ of clearance between the shive center and the ground. Logging is accomplished through a retractable roof opening with the wireline path nearly vertical from the spool to the boom shive then over and back down to the borehole. The large winch has a capacity to hold $15,000 \mathrm{ft}$ of 7 conductor, 15/32-inch wireline. The winch is hydraulically operated and is capable of line speeds from 1-200 ft per minute. The winch can pull line at $50 \mathrm{ft}$ per minute with $10,000 \mathrm{lb}$ of tension. The winch is rated for a maximum load of $20,000 \mathrm{lb}$. The winch air brake is rated at $10,000 \mathrm{lb}$ line tension with the drum full and 14,000 lb with the drum empty and the cable fully extended. The mast, stabilizers, and outriggers are hydraulically operated and are rated at 14,000 lb of line pull with a safety factor of 3 applied to all components in the line-pull path.

The boom mast includes an integral 20,000-1b capacity loadcell and an encoder that resolves one turn of the shive into 512 increments, giving a position accuracy of about a tenth of an inch.

The truck has ac and dc lighting, air conditioning, and heating throughout. Power can be supplied by a 3-phase, $20-\mathrm{kV} \cdot$ A generator that is mounted below the logging cab of the truck.

\section{Transmitter Truck}

Figure 5 is a flowchart showing the transmitter truck's data links; three are active links with the receiver truck: the bidirectional voice communication link, the ethernet link, and the one-directional dual-analog signal-transmission links; all of which 


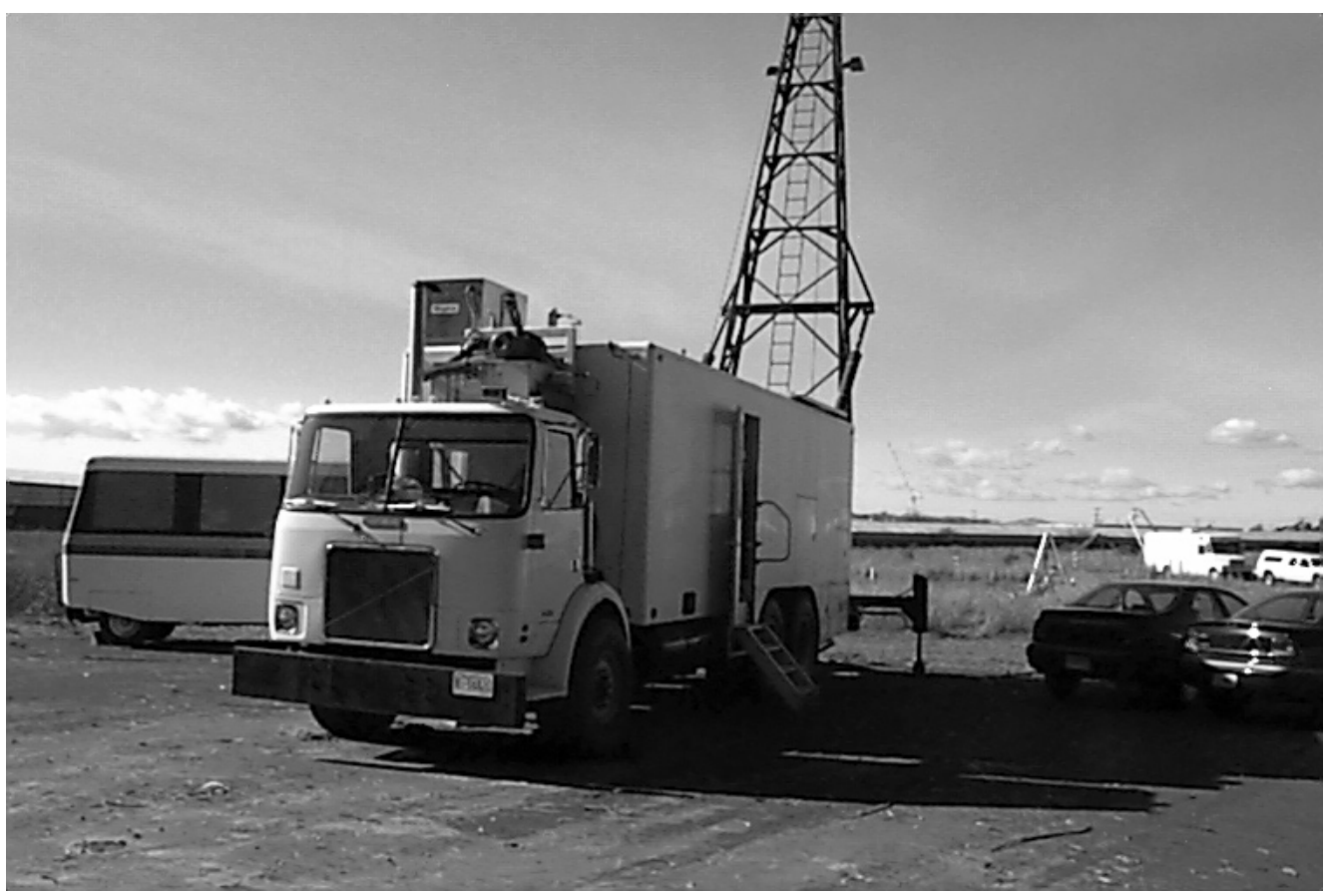

Figure 4. Logging mast fully extended on the receiver truck with the rear hydraulic stabilizer system activated. The large fuel capacity allows over $140 \mathrm{hr}$ of operation in remote field sites. The transmitter truck is shown to the far right of the photo-a separation distance of $52 \mathrm{~m}$.

are transmitted via fiber-optic cable to minimize inductive coupling with the system. The TCP/IP protocol is run under a software, or a virtual dataacquisition instrument developed from the LabVIEW software package, and is controlled by a Windows Pentium PC. The analog-signal-transmission lines carry information about the transmitter-coil depth and power to the receiver truck. The ethernet connection carries identical information, including the active TCP/IP link to the receiver truck; these ethernet data are used for separate calculations.

In crosswell electromagnetic imaging, highresolution phase information is necessary to determine geophysical parameters, and this requires accurate depth information. Both the receiver and transmitter trucks are equipped with optical encoders, which pulse with milliradian accuracy; these pulses are then converted to downhole distances, which are sent simultaneously to the PC and analog signal links.

The analog signal sent to the PC uses the counter input of one of two National Instruments NB MIO-16X E-50 analog-to-digital (A/D) cards. The second A/D card accepts the transmitter power signal, which comes from two Crown amplifiers configured in push/pull operation; this configuration effectively doubles the voltage so that enough current can travel through the $1800 \mathrm{ft}$ of cable to power the transmitter coil. The Crown amplifiers output an ac voltage proportional to the current driving the transmitter coil; spatial variance of ground conductivity partially determines the admittivity of the transmitter coil, and is thus necessary to monitor current change as a function of depth. The transmitter coil can be configured in two states: as an untuned LCR circuit for frequencies below $500 \mathrm{~Hz}$, and as a tuned resonant circuit for a specific frequency higher than $100 \mathrm{~Hz}$.

We are planning future improvements that include a general purpose interface bus (GPIB) connection, which controls the signal generator so that transmission signal modulation can be controlled from the receiver truck. Note that, in the current configuration, the transmitter PC displays real-time values of the transmitter depth and power so that the pulse modulation can be monitored in the transmitter truck.

As with the receiver truck, the transmitter truck was also procured from the Nevada Test Site. Previously, it was used as a general purpose field 


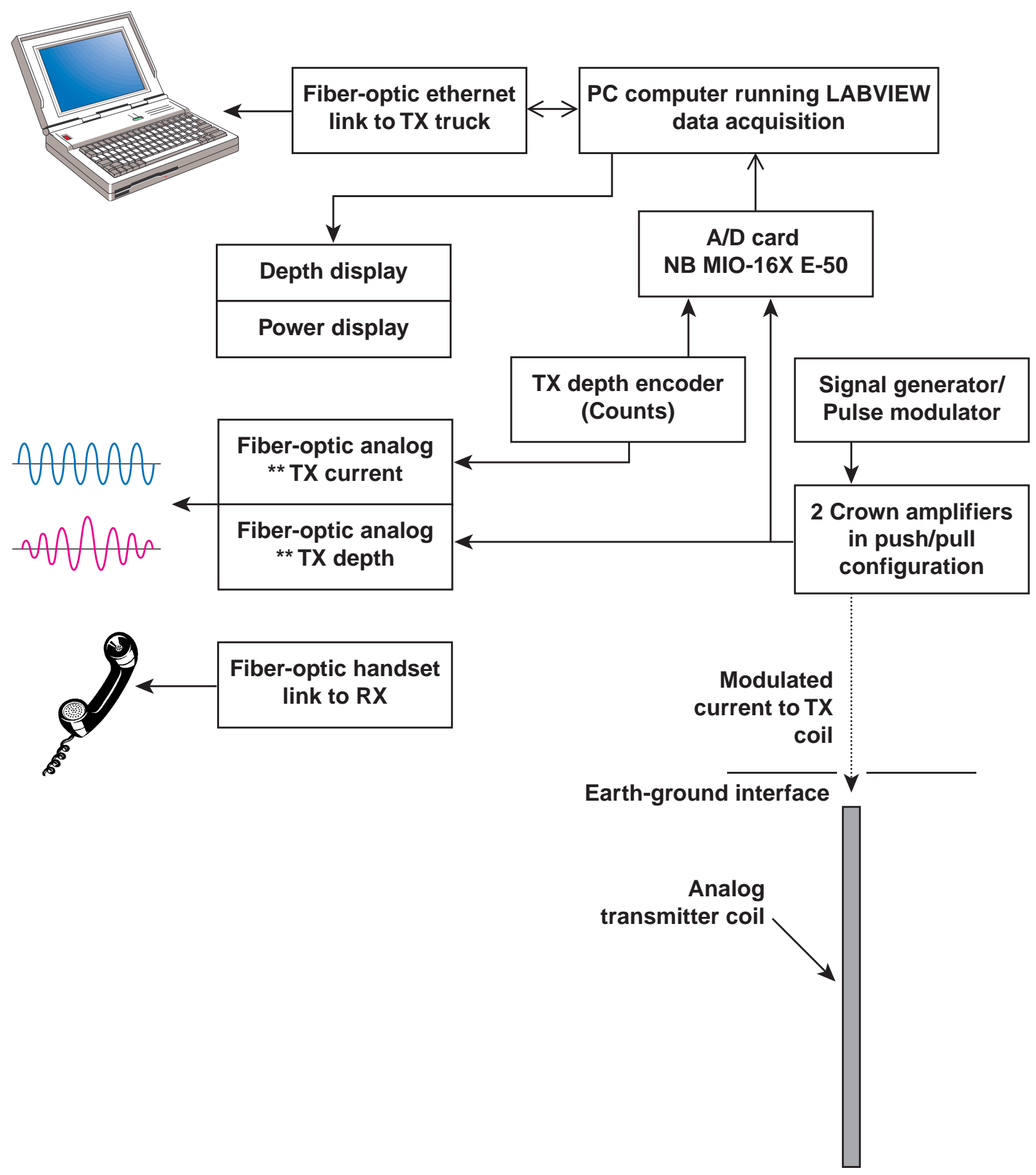

Figure 5. Flowchart of data links in the transmitter truck. Although future systems will use a GPIB interface to control the signal generator from the receiver truck, hydraulic control will remain under direct operator control. 


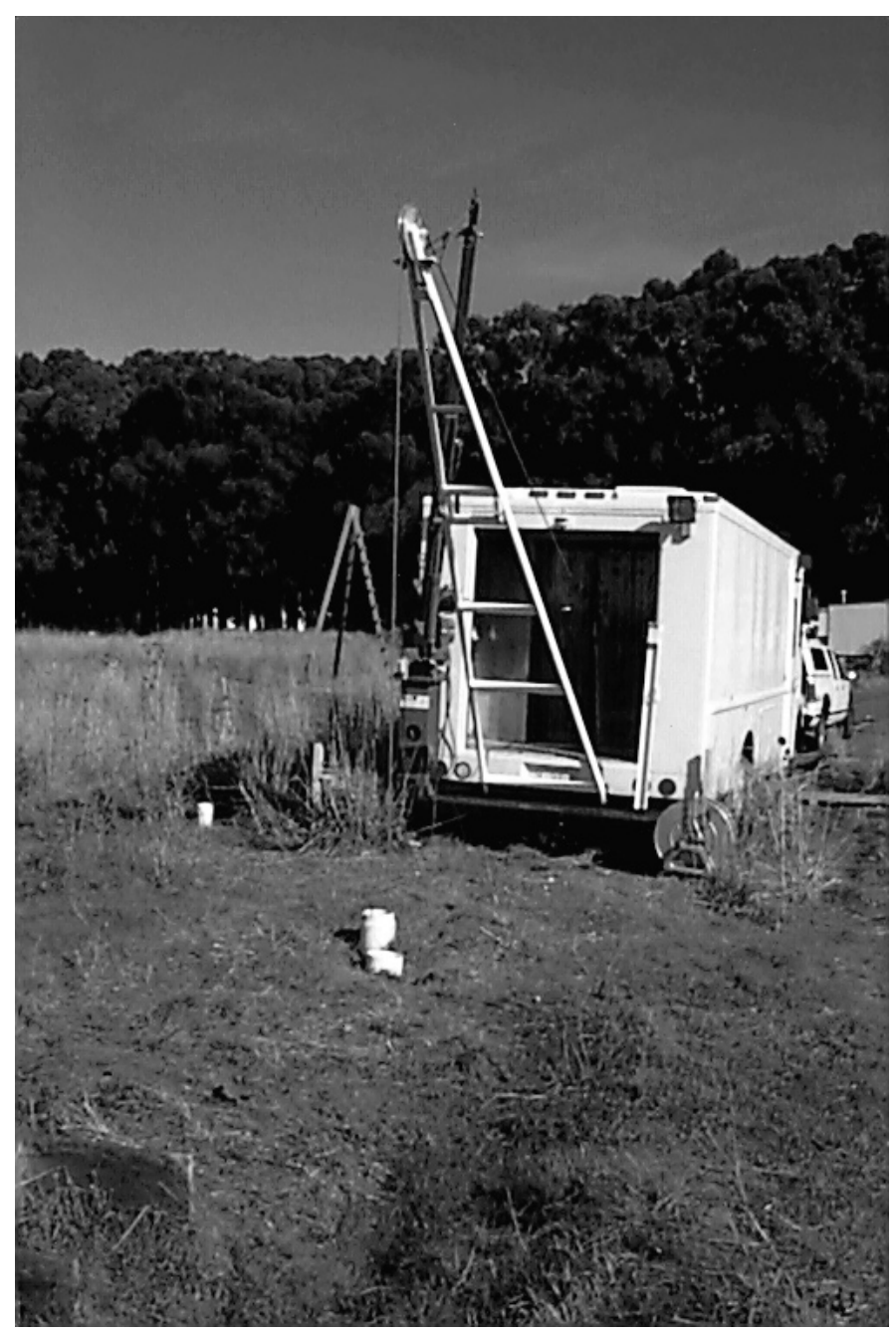

Figure 6. Rear view of the transmitter truck. The mast is controlled by a hydraulic boom on the port side of the truck. The borehole EMNW is located in the center of the picture.

vehicle (Figures 6 and 7). The truck is medium duty, with a 23,000-lb load rating.

We made major modifications to this vehicle so that it could serve as a transmitter platform. An integral A-frame boom was installed with $18 \mathrm{ft}$ of ground clearance. A hydraulically operated winch, with its associated hydraulic reservoir, was installed. The winch drum has capacity to hold $3500 \mathrm{ft}$ of 7 conductor 15/32-inch wireline. The winch is capable of wireline speeds between 10$100 \mathrm{ft}$ per minute and has a maximum tension rating of $5000 \mathrm{lb}$. The winch brake is rated at 7500-lb line tension with an empty drum. The winch also includes a load cell measuring up to $10,000 \mathrm{lb}$ pull and an encoder that divides one shive revolution into 512 increments. The position resolution is similar to the receiver truck, about $0.1 \mathrm{inch}$. The mast and outriggers are manually operated and are rated at a line pull of $10,000 \mathrm{lb}$ with a safety factor of 5 applied to all components in the line pull path.

We also mounted a general purpose crane on the truck's extended rear bumper. This crane has a telescoping arm and full three-axis range of motion. The crane is controlled by electric motors activated by the operator's push buttons. The crane is used to support the A-frame during logging operations or can be used for special lifting and lowering tasks.

The truck has ac and dc power throughout as well as heating and air conditioning; ac power is provided by a PTO-driven, one-phase, $8-\mathrm{kV} \cdot \mathrm{A}$ generator. 


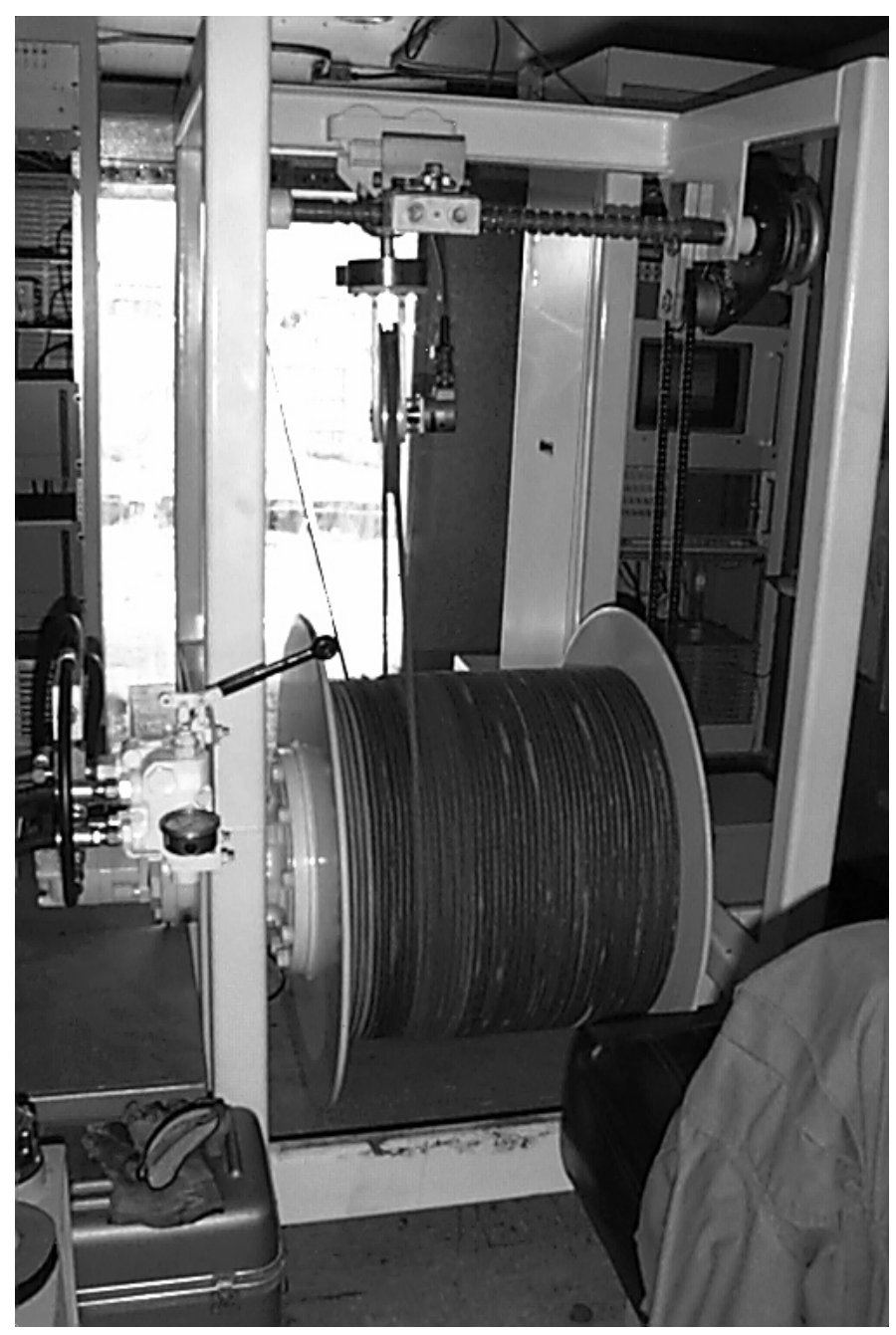

Figure 7. View from the inside of the transmitter truck. The winch in the center currently has $2400 \mathrm{ft}$ of cable, with a capacity expansion. The optical encoder is attached to the shive wheel above the center of the spool. Hydraulic controls are on the center left.

\section{Field Test Site}

The RFS, which is located on land managed by the University of California, provided an ideal site for testing our new data-acquisition field system and for determining the extent of coherency between known modeling code and field data. The field site and test holes are shown in Figure 8. This experiment utilized wells EMNW, a 70-m-deep plastic-cased hole, OBSS, a 30-m-deep plasticcased hole, and CAS1, a 30-m-deep steel-cased hole. Unequal well depth, as in this case, simply constrains our crosswell modeling attempts to the depth of the shallowest well. This experiment used well EMNW as the transmitter location and wells OBSS and CAS1 as the receiver locations. This geometry provided a steel-cased well within $10 \mathrm{~m}$ of a plastic-cased well for receiver locations, and a plastic-cased well at five times the distance for transmitter locations; this was a unique opportunity to examine induction through steel-cased wells. Although the RFS deployment acquired such data, the analysis includes the use and development of complex modeling procedures, which are beyond the scope of this paper.

The geological section of the RFS site can be divided into two primary units: a 40-m layer of unconsolidated deltaic deposits and a basement of sandstone or shale (Pouch, 1987). The unconsolidated deposits are primarily bay mud and clay with occasional layers of sand and gravel. One strata, at a depth of $30 \mathrm{~m}$, was the target zone for a 1993 saltwater injection experiment. Mostly pumped out in 1995, the residual saltwater left a small but significant resistivity signature. According to Pouch (1987), the resistivity of the clay ranges from 5 to $20 \mathrm{ohm}-\mathrm{m}$, whereas the sand and gravel ranges from 12 to $33 \mathrm{ohm}-\mathrm{m}$. 


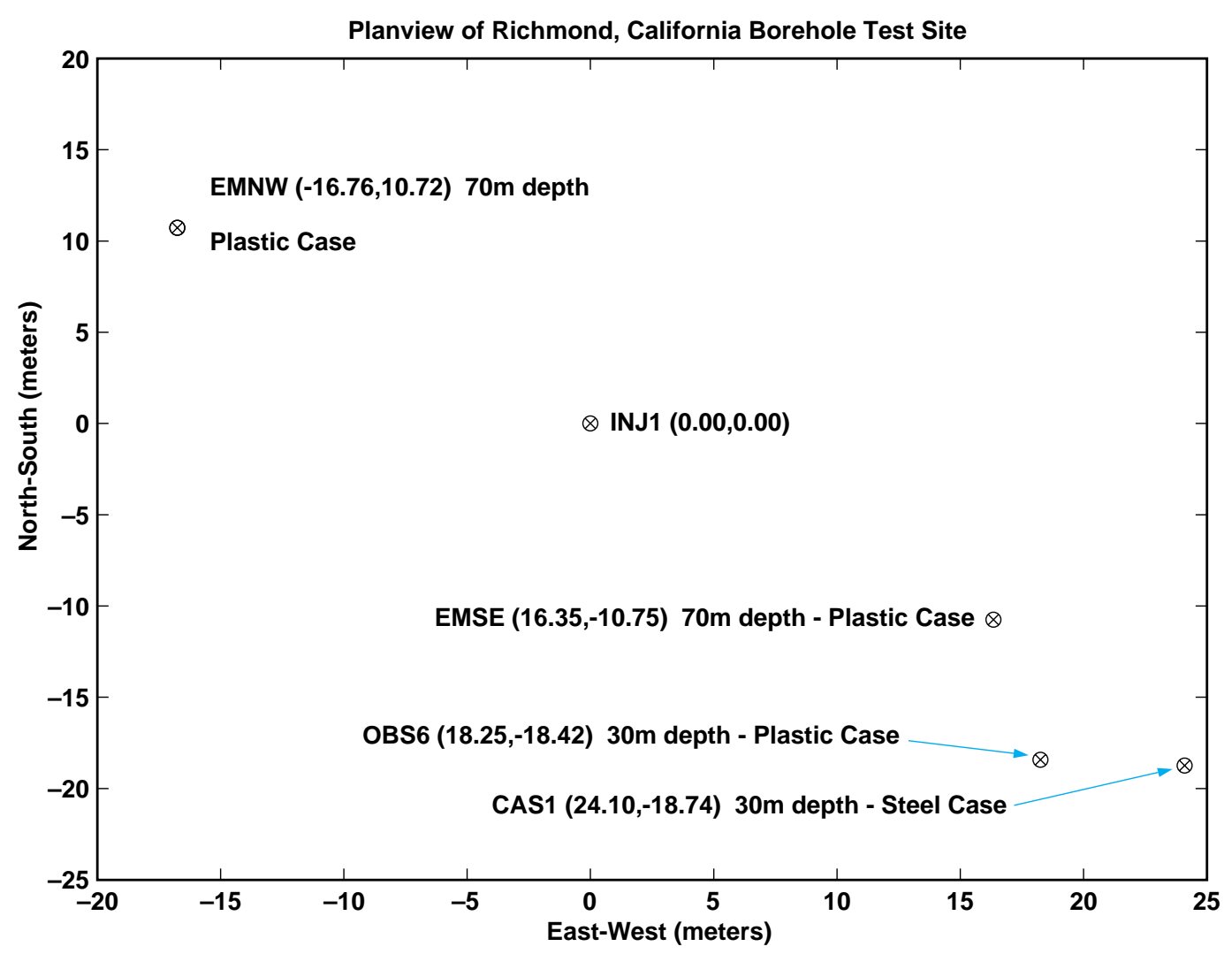

Figure 8. Planview of the Richmond Field Station. Well EMNW is used as the transmitter location and boreholes OBSS (plastic) and CAS1 (steel) are used for the receiver locations. Note the close proximity of wells OBSS and CAS1 in relation to EMNW; data acquired during this experiment will use this geometry in the analysis of the steel-cased-well scenario.

\section{Calibration Procedure}

Prior to interpretation, the receiver-coil voltage received at the lock-in amplifiers must be converted to an equivalent magnetic field due to a dipole of unit moment. This calibration procedure requires measuring the dipole moment of the transmitter and the phase correction factor for the complete crosswell system.

Since magnetic field intensity is assumed to be linearly proportional to the dipole moment, or strength, of the transmitter, the amplitude calibration is essentially the subtraction of this finite dipole moment to a unit moment. Ideally, the calibration setup would take place in the absence of terra firma (ground) since the ground affects the induction of the coil. One alternative is to use a modeling code to determine the effect of the ground on the measurements.

Calibration of the coils requires both the transmitter and receiver coils to be parallel and above the ground on nonmetallic platforms of a mea- sured height. Initially, the separation is $15 \mathrm{~m}$, and the current is just below saturation, approximately $20 \%$ of normal operating current. A stable reading of magnitude and phase is taken as the coils are increasingly separated until a $r^{1 / 3}$ magnetic field decay can be shown. At this distance, approximately $40 \mathrm{~m}$, the amplitude is increased to the normal operating current, producing a field proportional to the impedance of the driven system

$\frac{V_{\mathrm{RX}}}{I_{\mathrm{TX}}} \cdot k=\frac{100 \cdot M}{r^{3}}$,

where

$$
\begin{aligned}
& M=\text { magnetic moment of transmitter, } \\
& r=\mathrm{TX} / \mathrm{RX} \text { separation, } \\
& k=\text { calibration factor, } \\
& V_{R X}=\text { voltage at } \mathrm{RX} \text { coil, } \\
& I_{T X}=\text { source current. }
\end{aligned}
$$

Solving Eq. (1) for the unknown $k$ gives the calibration factor used to multiply each amplitude for unit dipole moment. 
The phase calibration uses the same phase measurements as the amplitude calibration method, but extrapolates the phase for zero offset. Physically, zero offset implies measurement that is physically impossible to achieve because of saturation. The zero-offset phase measurement is subtracted from the theoretical value of $180 \mathrm{deg}$ - for two parallel coils in air-and the result is the phase calibration factor.

\section{System Test Results}

\section{Data Reproducibility}

The RFS experiment allowed us to test the acquisition system in a number of ways; one of which was the repeatability measurements that offered a means to reveal any noise bias in the system. Results show that the phase and amplitude differences are less than $0.5 \mathrm{deg}$ and $0.6 \%$, respectively.

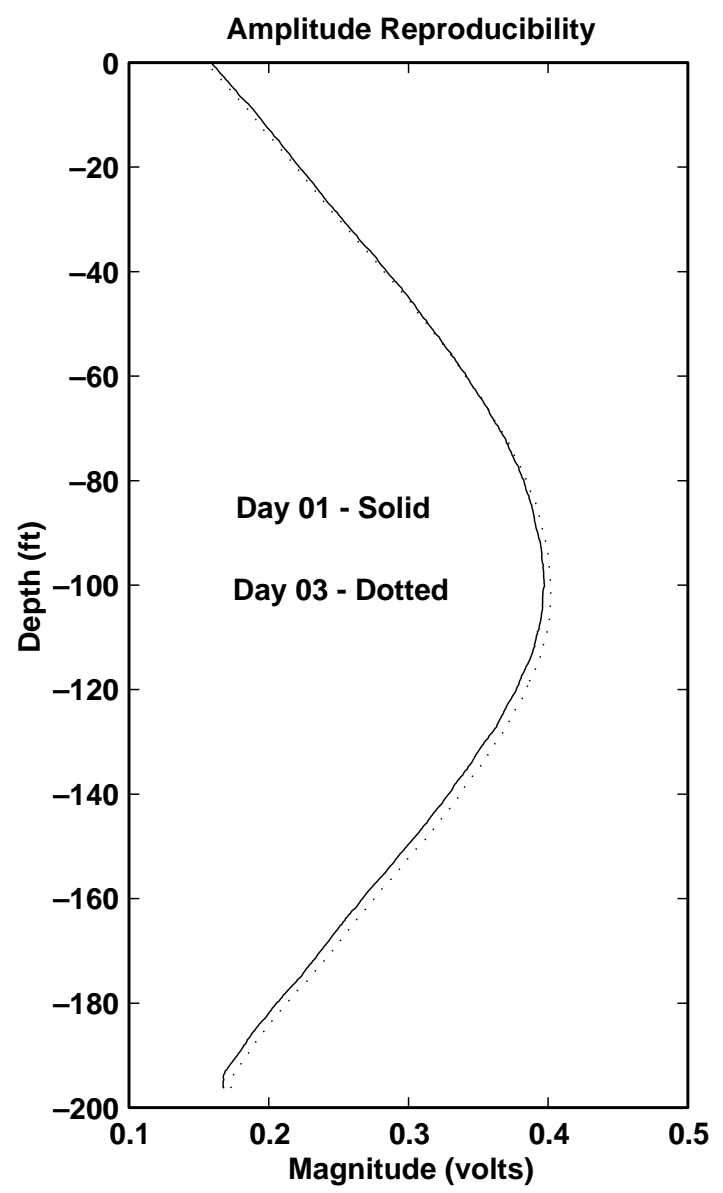

Figure 9 is a plot of the amplitude and phase of two equivalent profiles. The profiles are separated by two days and several setup and dismantling operations-a worst-case scenario. The phase discrepancy of approximately $0.5 \mathrm{deg}$ is attributed to temperature: the first profile (day 1) had outside temperatures of $43^{\circ} \mathrm{F}$, while the outside temperature during day 3 was $72^{\circ} \mathrm{F}$. Additionally, system power on day 3 was kept on until a steady-state thermal equilibrium occurred, while on day 1 , no system warm-up was allowed before repeatability measurements

\section{Modeled Crosswell Data}

One impetus for the RFS experiment was the potential to examine forward model coherency with field data. Forward models were run to match the field data to theoretical ground conditions. EM1D (Lee, 1986), a code that computes electromagnetic fields anywhere in an $\mathrm{N}$-layered earth,

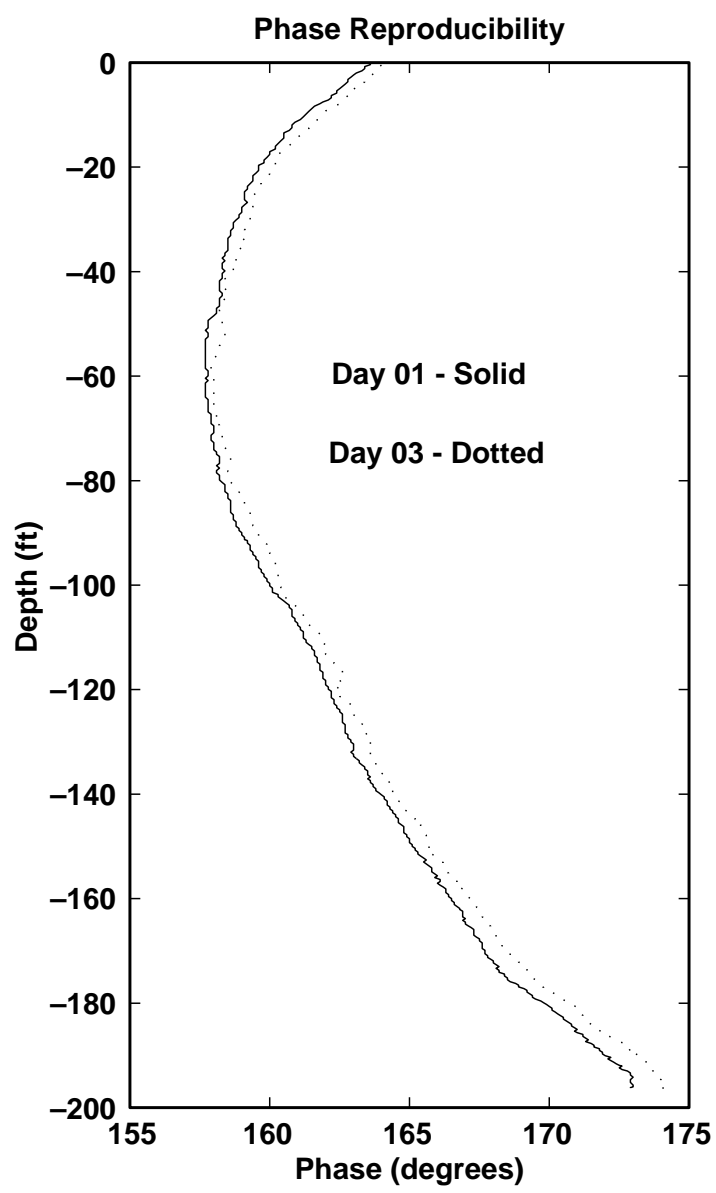

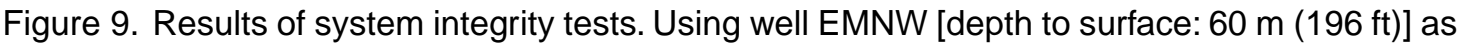

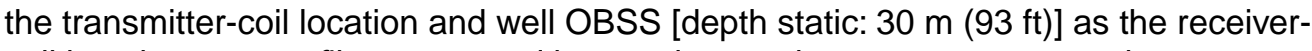
coil location, two profiles separated by two days under worst-case scenarios were acquired to ascertain reproducibility. 
was used to generate the dotted phase and amplitude models in Figure 10. In the earth model, a three-layered model, as described in Table 1, fits the phase quite accurately. Note, the next section describes information on well log versus forward model comparison.

Table 1: RFS model parameters.

\begin{tabular}{|c|c|c|}
\hline $\begin{array}{c}\text { Start depth } \\
(\mathrm{m})\end{array}$ & $\begin{array}{c}\text { End depth } \\
(\mathrm{m})\end{array}$ & $\begin{array}{c}\text { Resistivity } \\
(\mathrm{ohm}-\mathrm{m})\end{array}$ \\
\hline \hline 0.0 & 19.0 & 8.7 \\
\hline 19.0 & 36.5 & 9.2 \\
\hline 36.5 & $\infty$ & 22.2 \\
\hline
\end{tabular}

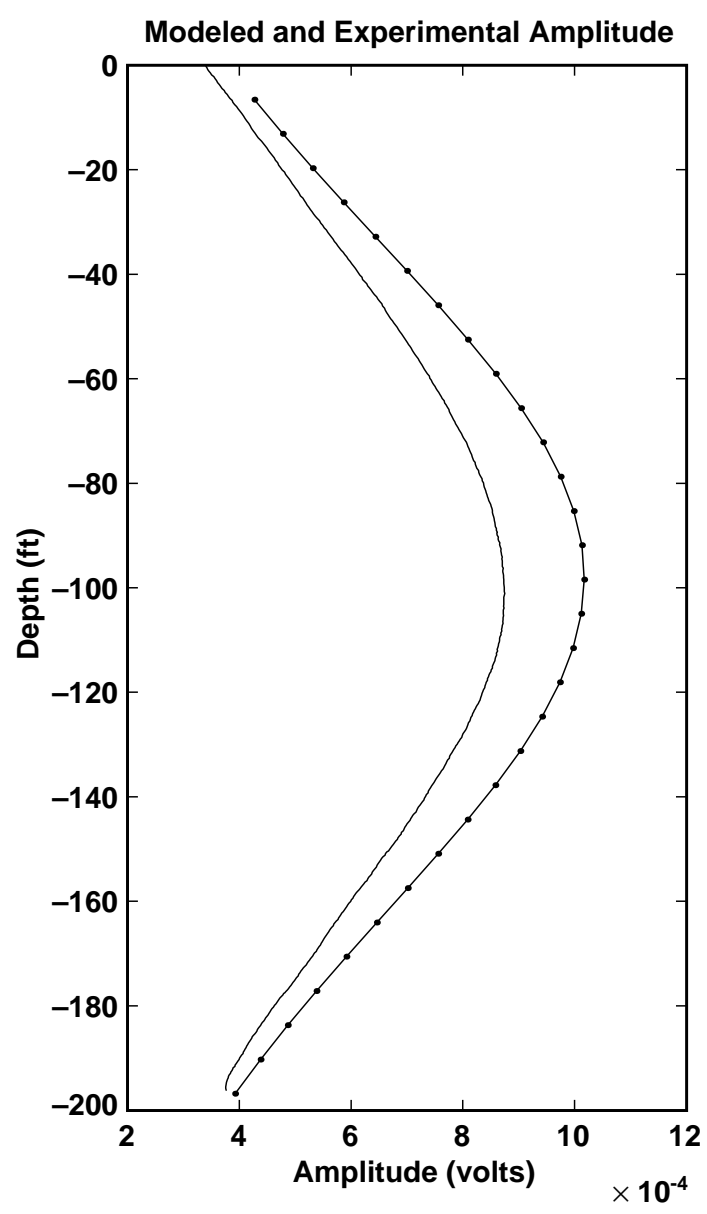

The phase is primarily a function of the layered conductivity and, therefore, is not expected to peak at the same depth. The amplitude model, however, has a maximum discrepancy of approximately $2 \times 10^{-4} \mathrm{~V}$, which is primarily due to error in the calibration process-most likely stemming from an error in the accepted value of the transmitter moment. Additional sources of error may also arise from the calibration process, when transmitter- and receiver-coil geometries are not accurately parallel.

Figure 11 plots the earth model found to optimally match the field data with the well log taken from the site in 1992. Immediately after the well $\log$ was taken, 55,000 gal of $1.0 \mathrm{~S} / \mathrm{m}$ saltwater was injected into a 3-m-thick aquifer at a depth of $30 \mathrm{~m}$. Since that time, pumping has removed a majority of the conductive water, although the residual

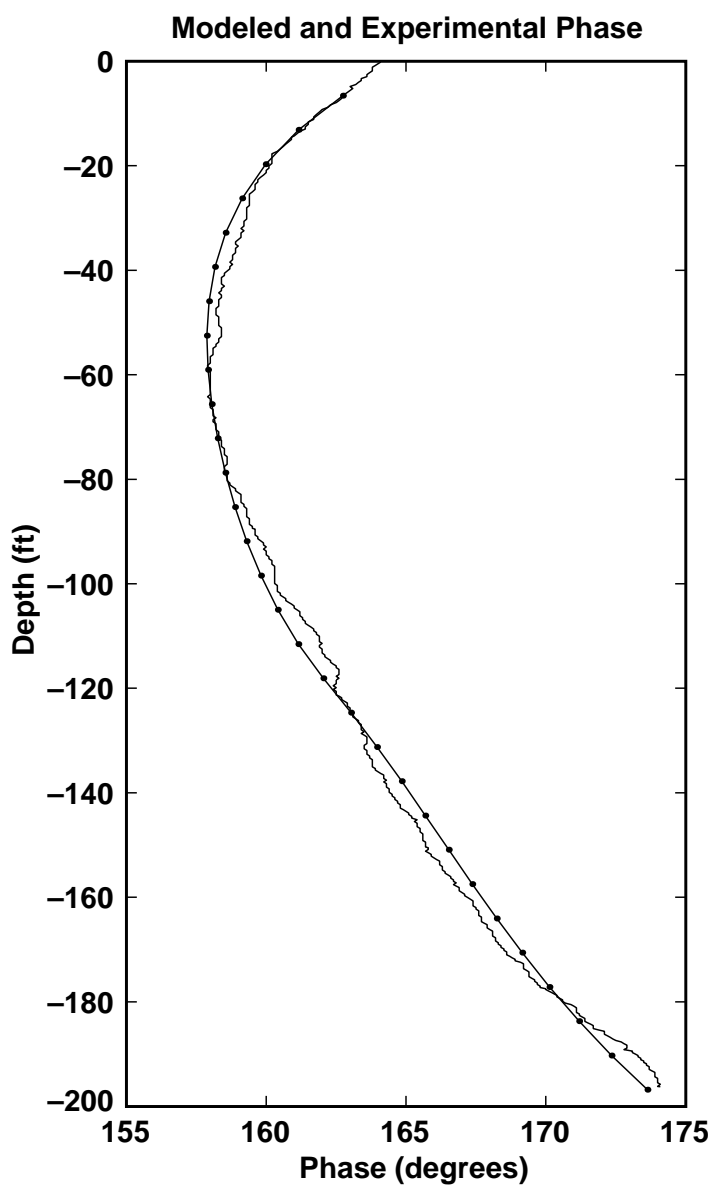

Figure 10. Comparison of EM1D forward model results (dotted) and acquired RFS field data (solid). The forward model was fit to the field data using a three-layered earth. See Table 1 for resistivity parameters as a function of depth. See Figure 11 for model comparison with well log data. The maximum amplitude discrepancy of $2 \times 10^{-4} \mathrm{~V}$ is due to a calibration error, primarily due to an error in the measured magnetic moment of the transmitter. The phase discrepancy is $0.5 \mathrm{deg}$ at the maximum, which is primarily due to system bias noise; this error is considered quite low. 

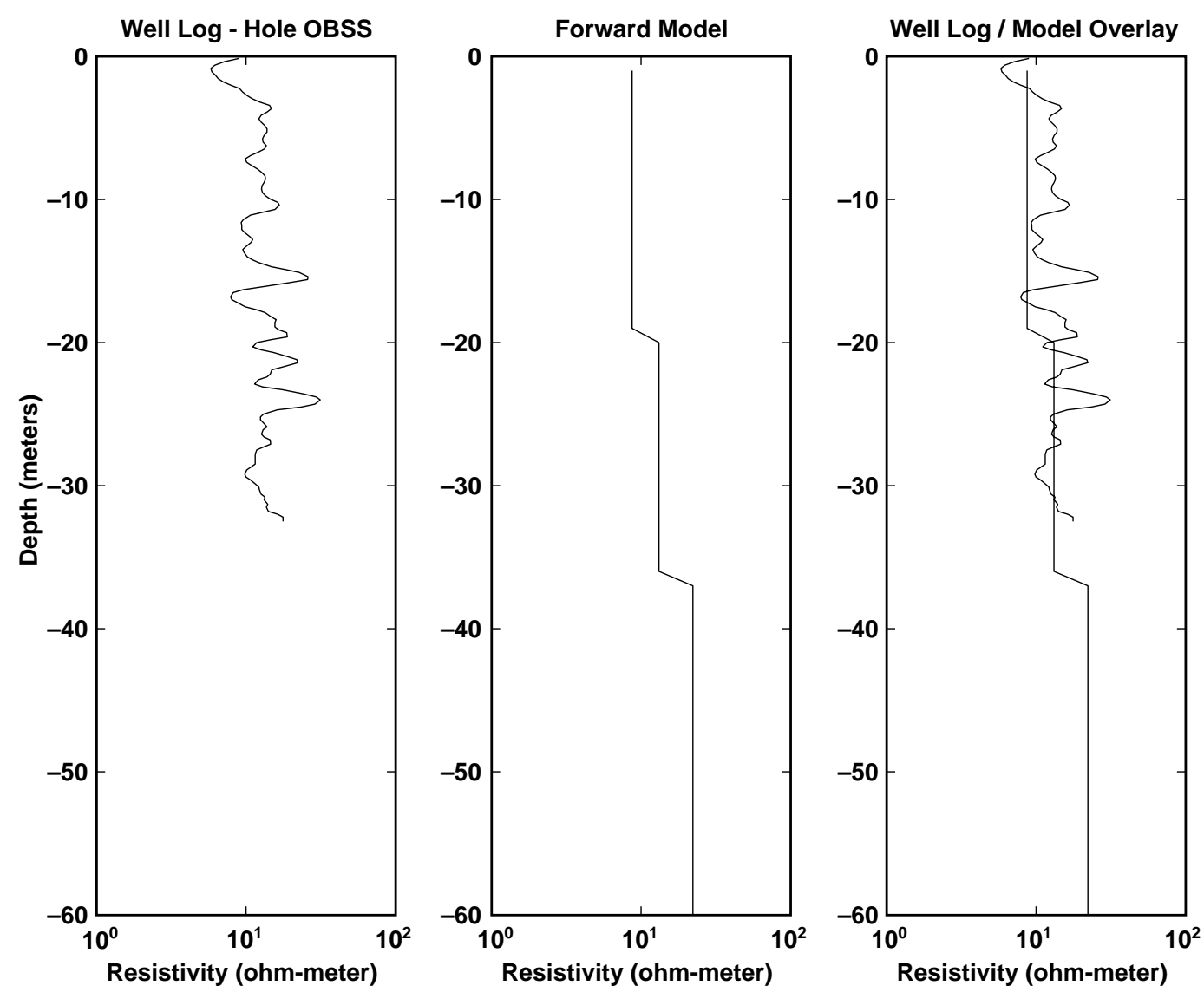

Figure 11. Comparison of well OBSS log data (left) to forward model (center). OBSS log data dates from 1993, acquired prior to a controlled spill; 55,000 gallons of $1.0 \mathrm{~S} / \mathrm{m}$ saltwater was injected into a 3-m-thick aquifer at a depth of $30 \mathrm{~m}$. In 1995, the salt plume was evacuated, although residual traces remain and are reflected in the several ohm-meter decrease offsets seen on the overlaid plot (right). Additionally, the forward model is extended to a depth of $60 \mathrm{~m}$, as the model response at shallow depths depends on lower depths

appears to have spread through tidal forces to shallow depths, causing a decrease in the resistivity at shallow depths, and a gap of several ohm-m between the earth model and well log.

\section{Inversion of Crosswell Data}

The earth model in Figure 11 suggests that inversion of this crosswell data set would be rather uneventful. Indeed, a two-dimensional inversion code, EMINV2D (Alumbaugh, 1993), applied to a set of profiles produced a resistivity contour with little variation. The shallow-hole depth of $30 \mathrm{~m}$ did not allow the imaging of bedrock that begins at a depth of $40 \mathrm{~m}$, and therefore, further inversion of these data were not pursued.

\section{Conclusion}

The crosswell low-frequency electromagnetic imaging program at LLNL has been upgraded to include two new borehole-logging field vehicles: one for signal transmission, and one for signal reception and data processing. In addition, a completely new and efficient data-acquisition system has been developed that relies on ethernet protocol to transfer information through fiber-optic cable.

The RFS site provided a location to test the reproducibility, background noise, and abilities of the system, and also to collect data concerning a unique well configuration; a steel-cased well and plastic-cased well in close proximity, such that we 
assume the formation between the two wells are identical. In addition, resistivity profiles were taken at the site and were matched with forward models. These models agree with well log data and show that the residual of a large saltwater plume injected into a 30-m depth strata early in 1993 caused a general resistivity decrease above a depth of $40 \mathrm{~m}$.

A rapid calibration procedure was introduced and tested; during this calibration process, the accepted value of the transmitter moment appears to be slightly in error. It will be necessary to determine the moment to a higher degree of accuracy.

\section{Future Research}

A significant portion of the data acquired at RFS concerns the steel-cased well scenario; typical oil-well sites have one fiberglass well for monitoring, with the remainder are cased in carbon steel. Therefore, the potential commercial application of crosswell electromagnetic imaging depends on the development of procedure and modeling code that deals with electromagnetic transmission through steel casing.

Crosswell electromagnetic imaging ultimately depends on the inversion technique, which in turn depends on the quantification of system noise and bias. It is widely known that ferromagnetic behavior is dependent on temperature changes. The characterization of the ferromagnetic induction core, the center of the transmitter and receiver coils, as a function of temperature is one such step toward controlling bias. In addition, controlled laboratory experiments in which the subsurface is well characterized should assist in the development and testing of inversion techniques appropriate to electromagnetic phenomena.

\section{Acknowledgments}

We gratefully acknowledge the contribution of Mike Wilt and EMI Inc. (Richmond, CA) for the use of high-quality testing equipment, field assistance, and insightful advice. Additionally, Don Lippert, Jim Doeherty, and John Clyde of Lawrence Berkeley National Laboratory and Frank Morrison of the University of California, Berkeley, contributed valuable time and information concerning assorted axioms of borehole recovery theory.

\section{References}

Alumbaugh, D.L., 1993, “Electromagnetic Conductivity Imaging with an Interative Born Inversion," IEEE Trans. Geoscience Remote, 31(4), 758763.

Lee, K.H., 1986, Program EM1D, Lawrence Berkeley National Laboratory, Berkeley, CA, fortran code, 1175 lines

Pouch, G.W., 1987, Hydrogeological Site Assessment of the Engineering Geoscience Well Field at the Richmond Field Station, Contra Costa County, California: M.Sc. thesis, Univ. of California at Berkeley, Berkeley, CA. 